\title{
Cost analysis between mini-percutaneous nephrolithotomy with and without vacuum-assisted access sheath
}

\author{
Elena Lievore ${ }^{1}$. Stefano Paolo Zanetti ${ }^{1}$. Irene Fulgheri ${ }^{2}$ - Matteo Turetti ${ }^{1}$. Carlo Silvani ${ }^{1}$. Carolina Bebi ${ }^{1}$. \\ Francesco Ripa ${ }^{1}$. Gianpaolo Lucignani ${ }^{1}$ - Edoardo Pozzi ${ }^{3}$. Lorenzo Rocchini ${ }^{1}$ - Elisa De Lorenzis ${ }^{1,4}$. \\ Giancarlo Albo $^{1,4}$. Fabrizio Longo ${ }^{1}$. Andrea Salonia ${ }^{3}$. Emanuele Montanari ${ }^{1,4}$. Luca Boeri ${ }^{1}$ (i)
}

Received: 1 July 2021 / Accepted: 5 August 2021 / Published online: 25 August 2021

(c) The Author(s) 2021

\begin{abstract}
Purpose To perform a cost analysis between vacuum-assisted percutaneous nephrolithotomy (vmPCNL) and minimally invasive PCNL (MIP) and explore potential predictors of costs associated with the procedures.

Methods We analyzed data from 225 patients who underwent vmPCNL or MIP at a single tertiary referral academic center between January 2016 and December 2020. We collected patients' demographics, peri-and postoperative data and detailed expense records. After propensity score matching, 108 (66.7\%) vmPCNL and 54 (33.3\%) MIP procedures were analyzed. Descriptive statistics assessed differences in clinical and operative parameters. Univariable and multivariable linear regression models tested the association between clinical variables and costs.

Results Operative time (OT) was shorter for vmPCNL, and the use of additional instruments to complete litholapaxy was more frequent in MIP (all $p \leq 0.01)$. Length of stay (LOS) was longer for MIP patients $(p=0.03)$ and the stone-free (SF) rate was higher after vmPCNL $(p=0.04)$. The overall instrumentation cost was higher for vmPCNL $(p<0.001)$, but total procedural costs were equivalent $(p=0.9)$. However, the overall cost for the hospitalization was higher for MIP than vmPCNL $(p=0.01)$. Univariable linear regression revealed that patient's comorbidities, OT, any postoperative complication and LOS were associated with hospitalization costs (all $p<0.001$ ). Multivariable linear regression analysis revealed that LOS and OT were associated with hospitalization costs (all $p<0.001$ ), after accounting for vmPCNL procedure, patients' comorbidities, and complications.

Conclusion vmPCNL may represent a valid option due to clinical and economic benefits. Shorter OT, the lower need for disposable equipment and the lower complication rate reduced procedural and hospitalization costs.
\end{abstract}

Keywords Percutaneous nephrolithotomy · Vacuum-assisted percutaneous nephrolithotomy · Cost analysis · Urolithiasis . Infectious complications

Luca Boeri

dr.lucaboeri@gmail.com

1 Department of Urology, IRCCS Foundation Ca' Granda, Ospedale Maggiore Policlinico, University of Milan, Via della Commenda 15, 20122 Milan, Italy

2 Department of Radiology, Foundation IRCCS Ca' Granda Ospedale Maggiore Policlinico, Milan, Italy

3 Division of Experimental Oncology/Unit of Urology, URI, IRCCS Ospedale San Raffaele, University Vita-Salute San Raffaele, Milan, Italy

4 Department of Clinical Sciences and Community Health, University of Milan, Milan, Italy

\section{Introduction}

Percutaneous nephrolithotomy (PCNL) is recommended as the standard procedure for large renal stones [1]. However, the introduction of miniaturized instrumentation has widened the indications of PCNL to a greater range of stone volumes [2]. Miniaturized PCNL was found to be as effective as standard procedure, but with decreased morbidity rates, bleeding, postoperative pain and shorter hospitalization $[3,4]$. On the contrary, major drawbacks of miniPCNL are longer operative time (OT), decreased visibility and higher intra-pelvic pressure, which is associated with postoperative infectious complications $[5,6]$. 
Recently, new technologies have been introduced to decrease the limitations of a smaller tract size. During standard PCNL, suction has been used mainly combined with ultrasound and ballistic devices to facilitate litholapaxy [7]. However, several Authors have proposed the use of various PCNL sheaths or nephroscopes equipped with suctioning mechanisms with promising outcomes [8-11]. Among the new instruments, the vacuum-assisted mini PCNL (vmPCNL) is a safe and effective treatment option for kidney stones $[12,13]$. Previous Authors have shown that vmPCNL was associated with shorter OT, reduced use of accessory devices for stone removal and lower intra-pelvic pressures than mini-PCNL, thanks to its continuous aspiration system that allows for simultaneous lithotripsy and litholapaxy [13, 14]. Consequently, the rate of infectious complications was lower for vmPCNL than classic PCNL $[13,14]$. Nonetheless, one of the major drawbacks of this system is its disposability, which may limit its use in the everyday clinical practice due to the fear of increasing the procedure-related costs.

We aimed to investigate clinical outcomes of patients with renal stones treated with vmPCNL or minimally invasive PCNL [11] at a single academic center and to perform a cost analysis between vmPCNL and MIP to explore potential predictors of costs associated with PCNL.

\section{Patients and methods}

\section{Study cohort}

We retrospectively analyzed all consecutive patients who underwent mini-PCNL for kidney stones at our tertiaryreferral academic center between January 2016 and December 2020. All procedures from 01/2016 to 08/2017 were performed with the Minimally Invasive PCNL Set (MIP) (Karl Storz \& Co. KG, Tuttlingen, Germany). Conversely, from 09/2017 to 12/2020 all mini-PCNL were carried out using the ClearPetra Set (Well Lead Medical Co., Ltd., China).

Patient's demographics and medical history were collected. Body mass index (BMI) $\left(\mathrm{kg} / \mathrm{m}^{2}\right)$ was calculated for every patient. Comorbidities were scored with the Charlson comorbidity index (CCI) [15]. For the specific purpose of this study, the CCI was categorized as $0 \mathrm{vs}$. $\geq 1$. A preoperative contrast enhanced computed tomography (CT) scan was requested. The stone volume was calculated using the ellipsoid formula (length $\times$ width $\times$ height $\times \pi \times$ 1/6) [16]. Preoperative bladder urine culture was required for each patient. Patients with negative bladder urine culture were treated with one-shot II generation cephalosporin before surgery (if not contraindicated) [17]. Patients with asymptomatic bacteriuria started a targeted therapy 48-72 h before PCNL. In cases of leukocytosis, urinary symptoms or fever, the surgery was postponed after a full antibiotic course and a subsequent negative bladder urine culture.

All procedures were performed by two experienced (> 150 PCNL performed) endourologists (E.M; F.L.) and the surgical technique was standardized for both surgeons [14].

\section{Surgical technique}

With the patient under general anesthesia, and placed in the supine Valdivia position, the procedure started with the placement of a ureteral catheter in the renal pelvis to inject contrast medium. Renal puncture was performed under combined fluoroscopic/ultrasonographic control and tract dilation was executed one-shot with the MIP $16 \mathrm{Ch}$ metallic dilator, or with the ClearPetra sheath assembled with its stylet. A $550 \mu \mathrm{m}$ Holmium: YAG laser fiber was used for stone fragmentation. Litholopaxy was performed by using the "vacuum-cleaner effect" during MIP, or through the aspiration-assisted sheath during vmPCNL. Flexible ureteroscope (7.9 Fr, Olympus URF-P6, Germany) and nitinol baskets were used through the percutaneous access when residual fragments could not be removed with the previous devices. An $8 \mathrm{Ch}$ nephrostomy tube was positioned as exit strategy in all cases, while the ureteral catheter was left in place at the end of the procedure only after non-stone-free or complicated procedures, based on the surgeon's preference.

\section{Intraoperative and postoperative data}

The litholapaxy modality and OT (defined as the time from ureteral catheter placement to exit strategy) were recorded. According to internal protocol of our institution, uncomplicated procedures were managed as follows: on postoperative day one the bladder catheter was removed and the nephrostomy tube was closed; on postoperative day two a percutaneous pyelography was performed to assess ureteral canalization and the presence of residual stones. If ureteral canalization was confirmed, the nephrostomy tube was removed. Patients were discharged on postoperative day three.

Postoperative complications were graded according to the PCNL-adjusted Clavien Score [18]. Blood cultures were collected in case of fever (body temperature $\geq 38{ }^{\circ} \mathrm{C}$ ) and/ or chills after surgery. Postoperative antibiotic treatment was decided after consultation with the Infectious disease department.

Patients were evaluated within 3 months after surgery with abdominal ultrasound (US) or CT scan to identify residual stones. The stone free (SF) status was defined as the absence of residual fragments $>4 \mathrm{~mm}$ in diameter. 


\section{Cost analysis}

The accounts department of the hospital provided detailed expense reports, which were used to compare hospital costs for each procedure. We recorded the cost of surgical instruments, operation facilities, medications, laboratory services, radiology tests and any additional procedure that was performed for surgery-related complications (e.g. CT scan, angioembolization, placement of ureteral catheter). The operating fee was calculated using the operative time per the cost of the operative room equipe (two surgeons, one anesthesiologist, two nurses, one radiographer). The total procedural costs were calculated as the sum of the costs for the employment of the operative room (operating fee) and surgical instruments. The total hospitalization costs were calculated as the sum of the costs of overall hospitalization (procedural costs, laboratory, radiology, pharmacy, room and board) thus, including complication-related costs with any additional procedures, including readmission due to major complication and related procedures. Costs of instruments did not change in the study period. Common costs, including preoperative and postoperative visit costs, were not included in this study as routine management of PCNL patients.

A cohort of 225 patients who underwent miniPCNL (including vmPCNL and MIP) between 01/2016 and $12 / 2020$ was identified. We excluded patients with congenital renal anomalies $(N=11)$; scheduled staged procedures for large stone burden $(N=30)$; concomitant additional procedures $(N=12)$; endoscopic combined intrarenal surgery (ECIRS) procedures $(N=2)$; stone fragmentation performed with ballistic, ultrasound or combined modality $(N=22)$; some patients presented more than one exclusion criteria. A sample of $120(65.9 \%)$ and $62(34.1 \%)$ patients treated with vmPCNL and MIP with complete perioperative and followup data was considered for statistical analyses.

Data collection followed the principles outlined in the Declaration of Helsinki. All patients signed an informed consent agreeing to share their own anonymous information for future studies. The study was approved by the Foundation IRCCS Ca' Granda-Ospedale Maggiore Policlinico Ethical Committee (Prot. 25508).

\section{Statistical methods}

We performed 1:2 propensity-score matching (nearestneighbor analyses using a caliper width of 0.2 of the standard deviation of the logit of the propensity score) to control for measurable baseline differences among patients in the two groups [19]. Propensity scores were computed by modeling logistic regression with the dependent variable as the odds of being in the MIP group and the independent variables as age, BMI, CCI, stone volume, and stone location. After matching, 108 (66.7\%) and 54 (33.3\%) individuals in the vmPCNL and MIP group, respectively, were considered for the final analysis.

Distribution of data was tested with the Shapiro-Wilk test. Data are presented as medians (interquartile range; IQR) or frequencies (proportions). After matching, descriptive statistics were used to assess potential differences in terms of clinical parameters, intraoperative and postoperative characteristics between the MIP and the vmPCNL group. The statistical significance of differences in medians and proportions were tested with the Mann-Whitney $U$ test and Fisher's exact test, as indicated.

Univariable and multivariable linear regression models tested the association between clinical variables and total hospitalization costs in the whole cohort. Statistical analyses were performed using SPSS v.26 (IBM Corp., Armonk, NY, USA). All tests were two sided, and statistical significance level was determined at $p<0.05$.

\section{Results}

Before matching, stone volume [median (IQR) 2.6 (1.8-3.0) vs. $\left.1.7(1.2-3.4) \mathrm{cm}^{3} ; p=0.01\right]$ was higher in the MIP than the vmPCNL group. There was also a significant difference in stone location between groups before matching $(p=0.03)$. After matching, patients and perioperative characteristics were evenly distributed.

Table 1 details descriptive statistics of the whole cohort according to the type of surgery after matching. OT was shorter during vmPCNL than MIP procedures [89 (73-126) vs. 115 (90-160) $\min ; p<0.001]$. The use of flexible ureteroscopes and baskets to complete litholapaxy was more frequently reported during MIP than vmPCNL (all $p \leq 0.01$ ). Hospital stay was longer after MIP than vmPCNL procedures $(p=0.03)$ and the SF rate was higher after vmPCNL than MIP $(p=0.04)$.

The rate of infectious complications was higher after MIP than vmPCNL $(24.0 \%$ vs. $8.3 \%, p<0.01)$ (Table 2$)$. Conversely, rates of overall postoperative complications were similar between groups (38.5\% for the MIP vs. $24.1 \%$ for vmPCNL; $p=0.07)$. Clavien-Dindo grade $>$ II complications were found in $7(6.4 \%)$ and $4(7.4 \%)$ patients after vmPCNL and MIP $(p=0.3)$, respectively (Table 2$)$.

Table 3 reports the basic equipment for each procedure and related costs. The overall cost for the surgical instruments of PCNL procedure was higher for vmPCNL than MIP $(p<0.001)$. Supplementary Table 1 depicts costs associated with additional equipment, laboratory or radiologic tests and procedures.

The cost-analysis of vmPCNL and MIP is shown in Table 4. Total procedural costs were equivalent between groups [999.7 (922.1-1158.4) $€$ for MIP and 1000.7 $(924.7-1161.3) €$ for vmPCNL, $p=0.9]$. Conversely, costs 
Table 1 Demographic characteristics and descriptive statistics of patients according to the type of surgery after matching

\begin{tabular}{|c|c|c|c|}
\hline & vmPCNL & MIP & $p$ value* \\
\hline No. of individuals & $108(66.7 \%)$ & $54(33.3 \%)$ & \\
\hline Age (years) & & & 0.7 \\
\hline Median (IQR) & $56(50-75)$ & $56(50-76)$ & \\
\hline Range & $22-81$ & $23-82$ & \\
\hline Male Gender [No. (\%)] & $65(60.2)$ & $37(68.5)$ & 0.5 \\
\hline BMI $\left(\mathrm{kg} / \mathrm{m}^{2}\right)$ & & & 0.7 \\
\hline Median (IQR) & $25(23-27)$ & $25(22-28)$ & \\
\hline Range & $19-41$ & $19-40$ & \\
\hline $\mathrm{CCI} \geq 1[$ No. $(\%)]$ & $31(28.7)$ & $17(31.4)$ & 0.6 \\
\hline Laterality [No. (\%)] & & & 0.8 \\
\hline Right & $48(44.5)$ & $23(42.6)$ & \\
\hline Left & $60(55.5)$ & $31(57.4)$ & \\
\hline Stone volume $\left(\mathrm{cm}^{3}\right)$ & & & 0.5 \\
\hline Median (IQR) & $2.2(1.1-3.7)$ & $2.2(1.1-3.5)$ & \\
\hline Range & $0.6-19.0$ & $0.5-19.0$ & \\
\hline Single stone $[$ No. $(\%)]$ & $43(39.8)$ & $22(40.7)$ & 0.8 \\
\hline Stone location & & & 0.3 \\
\hline Upper pole calices & $17(15.7)$ & $9(16.6)$ & \\
\hline Mid pole calices & $38(35.1)$ & $20(37.1)$ & \\
\hline Lower pole calices & $69(63.8)$ & $32(59.3)$ & \\
\hline Pelvis & $43(39.8)$ & $25(46.2)$ & \\
\hline Staghorn stone [No. (\%)] & $34(31.5)$ & $17(31.4)$ & 0.6 \\
\hline Stone density (Hounsfield unit) & & & 0.5 \\
\hline Median (IQR) & $1034(891-1501)$ & $1041(743-1444)$ & \\
\hline Range & $176-2290$ & $460-2032$ & \\
\hline Operative time (min) & & & $<0.001$ \\
\hline Median (IQR) & $89(73-126)$ & $115(90-160)$ & \\
\hline Range & $35-210$ & $60-255$ & \\
\hline Litholapaxy with basket [No. (\%)] & $42(38.8)$ & $50(92.6)$ & $<0.001$ \\
\hline Use of flexible ureteroscope [No. (\%)] & $44(40.7)$ & $33(61.1)$ & $<0.01$ \\
\hline Exit strategy [No. $(\%)]$ & & & 0.1 \\
\hline Nephrostomy only & $83(76.8)$ & $35(64.8)$ & \\
\hline Nephrostomy + Ureteral catheter & $25(23.2)$ & $19(35.2)$ & \\
\hline Hospitalization time (days) & & & 0.03 \\
\hline Median (IQR) & $4(3-5)$ & $5(3-6)$ & \\
\hline Mean (SD) & $4.2(3.1)$ & $5.8(3.7)$ & \\
\hline Range & $2-12$ & $2-14$ & \\
\hline Hemoglobin drop (g/dL) & & & 0.2 \\
\hline Median (IQR) & $-1.5(-1.9$ to -0.9$)$ & $-1.6(-2.8$ to -0.7$)$ & \\
\hline Range & -5.1 to -0.1 & -6.0 to -0.2 & \\
\hline Stone free rate $[$ No. $(\%)]$ & $98(90.7)$ & $42(79.6)$ & 0.04 \\
\hline Auxiliary procedures [No. $(\%)]$ & & & 0.08 \\
\hline No treatment & $6(5.5)$ & 7 (12.9) & \\
\hline RIRS & $2(1.9)$ & $2(3.7)$ & \\
\hline Second look PCNL & $2(1.9)$ & $3(5.5)$ & \\
\hline
\end{tabular}

$v m P C N L$ vacuum-assisted miniPCNL, $M I P$ minimally invasive PCNL, BMI body mass index, $C C I$ Charlson Comorbidity Index, $P C N L$ percutaneous nephrolithotomy, RIRS retrograde intrarenal surgery;

${ }^{*} p$ value according to the Mann-Whitney $U$ test and Fisher's exact test, as indicated 
Table 2 Postoperative complications in the whole cohort after matching $($ No. $=162)$

\begin{tabular}{lllc}
\hline & vmPCNL $(N=108)$ & MIP $(N=54)$ & $p$ value* \\
\hline $\begin{array}{l}\text { Overall complications [No. (\%)] } \\
\text { Highest Clavien-Dindo [No. (\%)] }\end{array}$ & $26(24.1)$ & $21(38.8)$ & 0.07 \\
$\quad$ I-II & $19(17.6)$ & $17(31.4)$ & 0.3 \\
IIIa-IIIb & $7(6.4)$ & $4(7.4)$ & \\
Blood transfusion [No. (\%)] & $1(0.9 \%)$ & $2(3.7 \%)$ & 0.2 \\
Infectious complications & & & \\
Any Clavien-Dindo [No. (\%)] & $9(8.3)$ & $13(24.1)$ & 0.01 \\
Readmission [No. (\%)] & $4(3.7)$ & $2(3.7)$ & 0.9 \\
Hemothorax & 1 & 0 & \\
Urine leakage & 1 & 0 & \\
Hematuria & 2 & 2 & \\
\hline
\end{tabular}

$v m P C N L$ vacuum-assisted miniPCNL, MIP Minimally invasive PCNL, PCNL Percutaneous nephrolithotomy

${ }^{*} p$ value according to the Fisher Exact test related to antibiotics $(p<0.01)$ and additional radiologi$\mathrm{cal} /$ laboratory examinations for the postoperative management of complications were higher for MIP than vmPCNL (all $p \leq 0.02$ ). The cost for in-hospital complications was higher for MIP than vmPCNL [mean (SD) 89.3 (50.3) $€$ vs. 34.8 (27.6) $€ ; p<0.01$ ]; similarly, the overall cost for the hospitalization was higher for MIP than vmPCNL [2658.2 (2084.4-3143.1) € vs. 2302.9 (1976.1-2693.1) $€ ; p=0.01]$. After discharge, 2 (3.7\%) and 4 (3.7\%) patients in the MIP and vmPCNL group, respectively, were re-admitted due to PCNL-related complications $(p=0.9)$. Specifically, 2 patients in the MIP group were readmitted for postoperative hematuria; conversely, hemothorax $(N=1)$, urine leakage $(N=1)$ and hematuria $(N=2)$ were causes for readmission after vmPCNL (Table 2). Costs associated with hospital readmission were similar between groups (Table 4).

Table 5 depicts linear regression models testing the association between clinical variables and total costs of hospitalization. Univariable linear regression models revealed that $\mathrm{CCI} \geq 1$, OT, the presence of postoperative complications (any), and length of stay (LOS) were all significantly associated with total hospitalization costs (all $p<0.001$ ). Conversely, vmPCNL was associated with a reduced hospitalization cost, compared to MIP (beta - 411.4€; 95\% CI $-697.1 €$ to $-125.9 € ; p<0.01)$. Multivariable linear regression analysis revealed that each day of hospitalization contributed $334.2 €(95 \%$ CI $302.4 €-365.9 € ; p<0.001)$ to the overall cost model, while for one hour increase in OT, the total hospitalization cost will increase by $234 €$ (95\% CI $144 €-330 €, p<0.001)$. Postoperative complications, CCI and the type of surgery were adjusted for in the cost model but did not affect total hospitalization costs.

The cost model suggested that a savings of approximately 1 day of hospitalization is required to offset the costs associated with the use of vmPCNL, as shown in the linear regression plots comparing hospitalization cost by LOS, stratified by surgical approach (Fig. 1). The point of cost equivalence for a vmPCNL case staying approximately 4 days is between 5 and 6 days with the MIP approach. Similarly, Fig. 2 represents the linear regression model showing the association between OT and hospitalization cost stratified by the two surgical techniques. The point of cost equivalence between vmPCNL and MIP was approximately $75 \mathrm{~min}$, after which vmPCNL was less costly than MIP procedures (Fig. 2).

\section{Discussion}

In this cross sectional study, we compared costs associated with vmPCNL and MIP procedures for kidney stones. We showed that, despite the additional cost of the disposable nephrostomic sheath, total procedural costs were comparable between groups, but total hospitalization costs were lower for vmPCNL than MIP.

The PCNL technique has evolved in the last decades, and one of the most recent advancement is the aspiration-assisted system, first introduced by Zeng et al. [20, 21]. High stone free rates, low complication rates, fast stone disintegration and shorter OT were all important characteristics of suction PCNL procedure [22]. vmPCNL, in particular, was associated with shorter OT, lower rates of infectious complications and lower need for additional equipment than classic miniPCNL [14]. However, there is a lack of reports that specifically investigated costs associated with suction assisted PCNL in the real-life setting.

Our study showed that, despite the overall cost for the surgical instruments of PCNL was higher for vmPCNL than MIP, the total procedural costs were comparable between groups. Indeed, vmPCNL was associated with 
Table 3 Basic equipment and related cost for PCNL procedures

\begin{tabular}{|c|c|c|c|}
\hline Instrument & $N$ & Cost for one unit (Euros) & $\begin{array}{l}\text { Cost for one } \\
\text { procedure } \\
\text { (Euros) }\end{array}$ \\
\hline \multicolumn{4}{|l|}{ General PCNL procedure } \\
\hline Sterile gynecological drape & 1 & 22.57 & 22.57 \\
\hline Nephroscopy surgical drape & 1 & 10 & 10 \\
\hline Medical camera drape & 2 & 1.07 & 2.14 \\
\hline Laser sterile drape & 1 & 0.8601 & 0.8601 \\
\hline $\mathrm{C}$-arm surgical drape & 1 & 35.883 & 35.883 \\
\hline Sterile surgical gowns & 5 & 3.6 & 18 \\
\hline Sterile surgical glove & 5 & 0.7564 & 3.782 \\
\hline 2-ways irrigation set & 1 & 1.1956 & 1.1956 \\
\hline Sterile suction tube & 1 & 1.444 & 1.444 \\
\hline Sterile fluid warming irrigation set & 1 (cost calculated for 4 uses) & 107.36 & 26.84 \\
\hline Sterile patient line irrigation set & 1 & 25.62 & 25.62 \\
\hline $20 \mathrm{ml}$ syringe & 6 & 0.0488 & 0.2928 \\
\hline $60 \mathrm{ml}$ syringe & 2 & 0.1326 & 0.2652 \\
\hline Foley bladder catheter ch 16 & 1 & 1.2078 & 1.2078 \\
\hline Antiseptic applicator $10.5 \mathrm{ml}$ & 2 & 3.41 & 6.82 \\
\hline Sodium chloride $0.9 \% 2000 \mathrm{ml}$ & 2 & 1.2031 & 2.4062 \\
\hline Sterile urine bag & 2 & 1.464 & 2.928 \\
\hline $1 \%$ lidocaine gel & 2 & 1.892 & 3.784 \\
\hline Hydrophilic guidewire & 2 & 24.278 & 48.556 \\
\hline Ultrasound probe drape & 1 & 5.49 & 5.49 \\
\hline Connector adapter & 1 & 5.002 & 5.002 \\
\hline Ureteral catheter ch 6 & 1 & 10.004 & 10.004 \\
\hline $8 \mathrm{ch}$ percutaneous nephrostomy set & 1 & 73.2 & 73.2 \\
\hline 0 silk suture & 2 & 0.7137 & 1.4274 \\
\hline Iopamidol 300-200 ml & 1 & 23.76 & 23.76 \\
\hline Laser fiber $550 \mu \mathrm{m}$ & 1 (cost calculated for 10 uses) & 888 & 88.80 \\
\hline \multicolumn{4}{|l|}{ MIP } \\
\hline Nephrostomy tract dilators & $1 \times 72$ procedures (yearly) & 195.58 & 5.42 \\
\hline \multirow[t]{2}{*}{ Nephrostomy sheath } & $1 \times 72$ procedures (yearly) & 542.08 & 15.06 \\
\hline & & Total for MIP & 441.99 \\
\hline \multicolumn{4}{|l|}{ vmPCNL } \\
\hline 16 Ch nephrostomy sheath & 1 & 256.2 & 256.2 \\
\hline \multirow[t]{2}{*}{ Stone collection bottle } & 1 & 10.12 & 10.12 \\
\hline & & Total cost for vmPCNL & 687.82 \\
\hline
\end{tabular}

PCNL Percutaneous nephrolithotomy, $v m P C N L$ vacuum-assisted miniPCNL, MIP Minimally invasive PCNL

shorter OT than MIP, which was the main factor determining the operating fee. Moreover, shorter OT may help limiting infectious complication by reducing the time span during which surgery is performed at elevated intrapelvic pressure. Aspiration-assisted mPCNL techniques have already been proved to be associated with less bleeding [10, 23], lower renal pelvic pressures and subsequent infectious complications $[6,12,14,24]$ than non-suctioning miniPCNL procedures. In this series we confirmed that infectious complications were lower for vmPCNL than MIP. Besides clinical implications, this finding had a relevant impact over the total hospitalization costs, thanks to the reduction of the expenses related to post-operative antibiotic therapies, radiological and laboratory tests and complication-related ancillary procedures. Infectious complications were associated with both greater hospitalization cost and increased LOS, supporting that cost savings with vmPCNL may be directly tied to expediting convalescence and reduced morbidity.

Similar to previous reports $[23,25]$ we observed a shorter LOS in the vmPCNL group than the MIP group. Consequently, the costs related to the hospital stay were 
Table 4 Cost analysis between vmPCNL and MIP (No. $=162)$

\begin{tabular}{|c|c|c|c|}
\hline Cost (Euros) & $\begin{array}{l}\text { vmPCNL } \\
(N=108)\end{array}$ & $\begin{array}{l}\text { MIP } \\
(N=54)\end{array}$ & $p$ value* \\
\hline Operating fee & & & $<0.001$ \\
\hline Median (IQR) & $253.8(211.5-352.5)$ & $338.4(310.2-447.6)$ & \\
\hline Range & $101.5-592$ & $169.2-724.7$ & \\
\hline \multicolumn{4}{|l|}{ Additional surgical equipment } \\
\hline Basket & & & $<0.001$ \\
\hline Median (range) & $62.2(0-162.5)$ & $162.5(0-325)$ & \\
\hline Nephrostomy tube & & & 0.2 \\
\hline Mean (range) & $6.5(0-6.7)$ & $12.1(0-67)$ & \\
\hline Total procedural costs & & & 0.9 \\
\hline Median (IQR) & $1000.7(924.7-1161.3)$ & 999.7 (922.1-1158.4) & \\
\hline Range & $790.1-1501.4$ & $679-1541.8$ & \\
\hline \multicolumn{4}{|l|}{ Post-operative costs } \\
\hline Antibiotic & & & $<0.01$ \\
\hline Median (IQR) & $3.3(3.2-13.1)$ & $8.2(3.8-27.5)$ & \\
\hline Range & $1.1-491.2$ & $1.6-316.4$ & \\
\hline Radiology test & & & $<0.01$ \\
\hline Mean (SD) & $6.7(4.3)$ & $24.9(15.7)$ & \\
\hline Range & $0.0-199$ & $0.0-455.4$ & \\
\hline Blood culture & & & 0.02 \\
\hline Mean (range) & $0.7(0-19)$ & $1.9(0-19)$ & \\
\hline Transfusion & & & 0.1 \\
\hline Mean (range) & $1.96(0-200)$ & $44.3(0-1350)$ & \\
\hline Additional procedures & & & 0.4 \\
\hline Mean (range) & $18.4(0-542.9)$ & $25.4(0-2370.9)$ & \\
\hline In hospital complications & & & $<0.01$ \\
\hline Mean (SD) & $34.8(27.6)$ & $89.3(50.3)$ & \\
\hline Range & $0.0-2394.9$ & $0.0-1805.4$ & \\
\hline Hospital stay & & & $<0.01$ \\
\hline Median (IQR) & $1200(900-1500)$ & $1500(1200-1800)$ & \\
\hline Range & $600-3600$ & $600-4200$ & \\
\hline Total hospitalization cost & & & 0.01 \\
\hline Median (IQR) & $2302.9(1976.1-2693.1)$ & $2658.2(2084.4-3143.1)$ & \\
\hline Range & $1546.4-7225.9$ & $1631.3-6235.9$ & \\
\hline Complications after discharge & & & 0.8 \\
\hline Mean (SD) & $204.3(129.5)$ & $172.8(152.5)$ & \\
\hline Range & $0.0-8942$ & $0.0-5327.1$ & \\
\hline
\end{tabular}

$v m P C N L$ vacuum-assisted miniPCNL, MIP Minimally invasive PCNL, PCNL Percutaneous nephrolithotomy, $O R$ Operative room

${ }^{*} p$ value according to the Mann-Whitney test significantly lower for vmPCNL and contributed to the lower total hospitalization costs of the vmPCNL compared to MIP.

The importance of this study is severalfold. First, it is the first report of a detailed cost analysis comparison between vmPCNL and MIP procedures. Second, we showed that vmPCNL was associated with a significantly shorter hospital stay, shorter OT and lower rate of perioperative complications compared to MIP, suggesting that expedited convalescence can compensate for the expenses associated with the disposable sheath. As reported by our linear model, by means of vmPCNL surgery approximately 1 day of hospitalization needs to be saved to make up for the costs associated with the disposable set. When examining cases where LOS was 4 days or less (the median hospitalization time for vmPCNL in our cohort), MIP was cheaper suggesting that cost equivalence is being primarily driven by lower perioperative morbidity and subsequent shorter LOS for vmPCNL. This was further confirmed by the multivariable 
Table 5 Linear regression models predicting total costs of hospitalization in the whole cohort after matching

\begin{tabular}{|c|c|c|c|c|c|c|}
\hline & \multicolumn{3}{|c|}{ UVA model } & \multicolumn{3}{|c|}{ MVA model } \\
\hline & Beta & $p$ value & $95 \% \mathrm{CI}$ & Beta & $p$ value & $95 \% \mathrm{CI}$ \\
\hline Age & 4.1 & 0.5 & -6.2 to 14.5 & & & \\
\hline $\mathrm{CCI} \geq 1$ & 297.3 & 0.04 & $7.9-586.7$ & 106.6 & 0.09 & -17.1 to 230.6 \\
\hline BMI & 13.1 & 0.4 & -19.6 to 45.6 & & & \\
\hline Stone volume & 20.5 & 0.6 & -27.9 to 68.8 & & & \\
\hline Operative time (min) & 8.2 & $<0.001$ & $5.1-11.3$ & 3.9 & $<0.001$ & $2.4-5.5$ \\
\hline Postoperative complications & 747.6 & $<0.001$ & $488.7-1006.4$ & 55.1 & 0.4 & -80.1 to 190.4 \\
\hline Hospitalization time (days) & 357.1 & $<0.001$ & $327.5-386.6$ & 334.2 & $<0.001$ & $302.4-365.9$ \\
\hline vmPCNL vs. MIP & -411.4 & $<0.01$ & -697.1 to -125.9 & -215.4 & 0.5 & -413.8 to 10.2 \\
\hline
\end{tabular}

UVA univariate model, $M V A$ multivariate model, $C C I$ Charlson Comorbidity Index, $B M I$ body mass index, $M I P$ minimally invasive PCNL, $C I$ confidence interval
Fig. 1 Smoothed linear regression analysis of length of stay versus hospitalization cost stratified by vacuum assisted PCNL (vmPCNL) (red) and minimally invasive PCNL (MIP) (green). Gray areas represent $95 \%$ confidence intervals. Vertical continuous and dashed lines display median and mean length of stay for each surgical technique, respectively. $P C N L$ Percutaneous nephrolithotomy

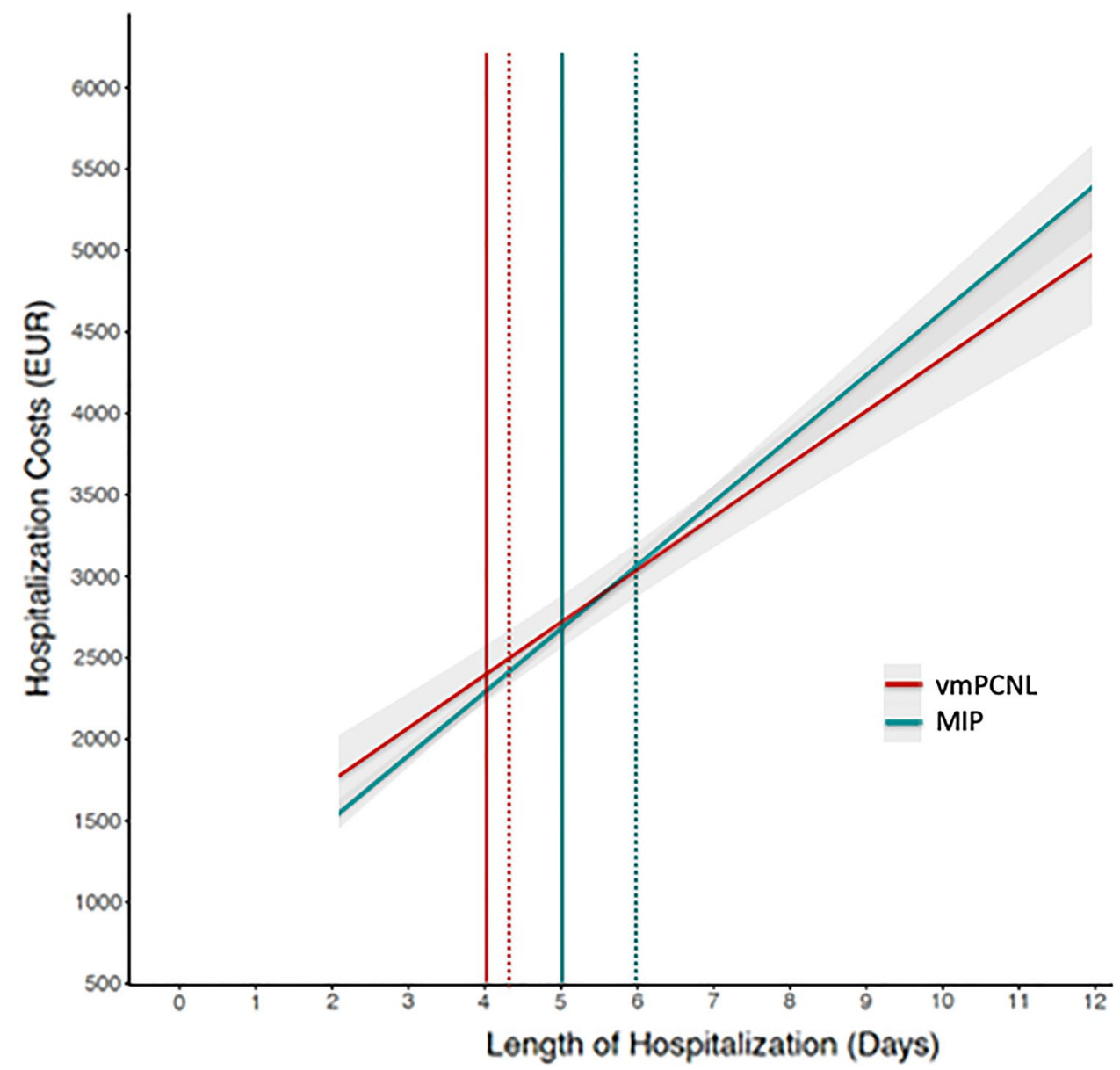

model where the effect of LOS and OT were independent from other factors known to influence hospitalization cost, thus including vmPCNL procedures.

In terms of OT the linear regression model suggests that, for procedure lasting more than $75 \mathrm{~min}$, vmPCNL is more cost-convenient than MIP. Adding this to the demonstrated clinical benefit of vmPCNL, in terms of lower rates of infectious complications and shorter hospital stay, it appears reasonable to privilege vmPCNL in cases in which a longer procedure is foreseen for stone's or patient's characteristics. However, despite being novel and innovative, these findings deserve validation from randomized clinical trials comparing aspiration and non-aspiration assisted mini-PCNL techniques.

Collectively, these findings imply that vmPCNL is potentially a cost equivalent option as MIP with faster 
Fig. 2 Smoothed linear regression analysis of operative time versus hospitalization cost stratified by vacuum assisted PCNL (vmPCNL) (red) and minimally invasive PCNL (MIP) (green). Gray areas represent $95 \%$ confidence intervals. Vertical dashed line displays the point of cost equivalence between the two groups. PCNL Percutaneous nephrolithotomy

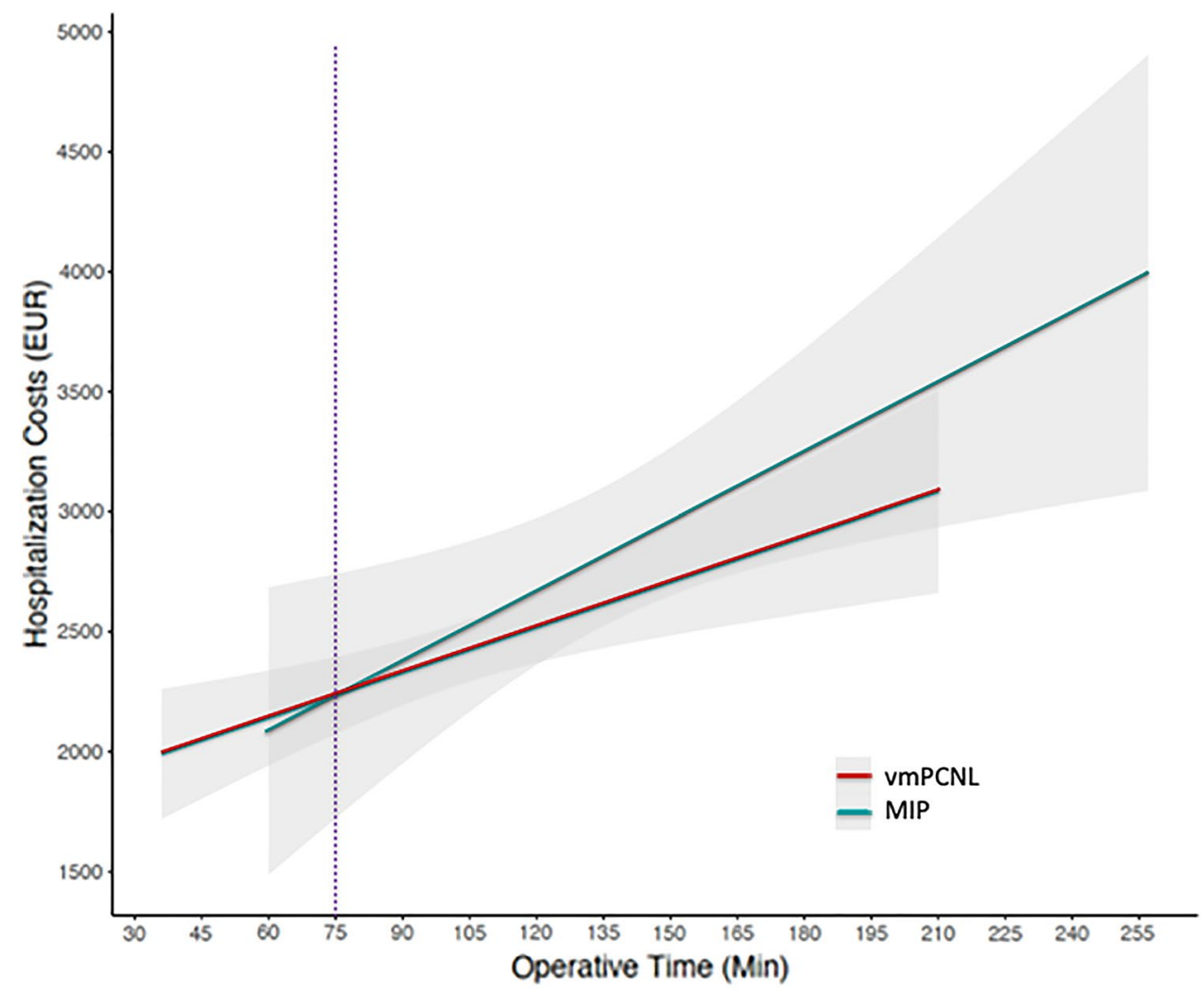

convalescence and minimal peri-operative morbidity in appropriately selected patients.

This study is not devoid of limitations. First, although propensity-score matching analysis is a solid method to reduce the selection bias of a retrospective study, the lack of randomization may limit the interpretation of our results. Second, this was a single center-based study, which raises the possibility of further selection biases Therefore, larger studies across different centers and cohorts are needed to externally validate our findings. Moreover, due to our internal protocol, median LOS was $>3$ days in this cohort, meanwhile hospitalization for uncomplicated PCNL is progressively shortening, especially in high volume centers [26]. Given the optimal results with these new technologies, we foresee a reduction of LOS, which may further confirm the feasibility of using single use surgical equipment. In addition, cost assessment might vary by hospital, in particular for high capital cost expenditures such as the disposable access sheath, which can be variably accounted for and in which cost-per-use will likely be lower at higher volume institutions. While this may limit applicability to any individual hospital/practice, we believe that our novel results should drive the organization of a population-based dataset that would be more representative of national outcomes compared to data from individual high-volume surgical centers.

\section{Conclusions}

While miniaturized PCNL remains the preferred treatment option for kidney stones, our findings suggest vmPCNL may represent an attractive option due to clinical and economic benefits. The shorter OT and the lower need for disposable equipment during vmPCNL surgery reduced the procedural costs and offset the use of the disposable set. Moreover, vmPCNL was characterized by lower total hospitalization costs due to the lower rate of infectious complications and associated costs (antibiotic therapies, radiology and laboratory tests, longer hospitalization). Future larger studies are needed to explore the true cost benefit of vmPCNL over classic miniaturized PNCL.

Supplementary Information The online version contains supplementary material available at https://doi.org/10.1007/s00345-021-03811-5.

\section{Acknowledgements None.}

Author contributions EL: Manuscript writing and editing, data collection; SPZ: Manuscript writing and editing, data management; IF: Data collection, project development; MT: Data collection; CS: Data collection; CB: Data collection; FR: Data collection; GL: Data collection; EP: Data analysis; LR: Data analysis; EDL: Data collection; GA: Data collection; FL: Data analysis; AS: Data analysis; EM: Project development; LB: Project development; data analysis; manuscript writing and editing. 
Funding Open access funding provided by Università degli Studi di Milano within the CRUI-CARE Agreement. None.

Availability of data and material None.

Code availability None.

\section{Declarations}

Conflict of interest The authors declare that they have no conflict of interest.

Ethical approval Data collection followed the principles outlined in the Declaration of Helsinki. The study was approved by the Foundation IRCCS Ca' Granda-Ospedale Maggiore Policlinico Ethical Committee (Prot. 25508).

Consent to participate All patients signed an informed consent agreeing to share their own anonymous information for future studies.

Consent for publication All patients signed an informed consent agreeing to share their own anonymous information for future studies.

Open Access This article is licensed under a Creative Commons Attribution 4.0 International License, which permits use, sharing, adaptation, distribution and reproduction in any medium or format, as long as you give appropriate credit to the original author(s) and the source, provide a link to the Creative Commons licence, and indicate if changes were made. The images or other third party material in this article are included in the article's Creative Commons licence, unless indicated otherwise in a credit line to the material. If material is not included in the article's Creative Commons licence and your intended use is not permitted by statutory regulation or exceeds the permitted use, you will need to obtain permission directly from the copyright holder. To view a copy of this licence, visit http://creativecommons.org/licenses/by/4.0/.

\section{References}

1. Türk C, Neisius A, Petř́ík A et al. (2021) EAU Guidelines on Urolithiasis. Eur Assoc Urol. https://uroweb.org/wp-content/uploads/ EAU-Guidelines-on-Urolithiasis-2021.pdf.

2. Zanetti SP, Talso M, Palmisano F et al (2018) Comparison among the available stone treatment techniques from the first European association of urology section of urolithiasis (EULIS) survey: do we have a queen? PLoS ONE 13(11):1-11. https://doi.org/10.1371/ journal.pone.0205159

3. Ruhayel Y, Tepeler A, Dabestani S et al (2017) Tract sizes in miniaturized percutaneous nephrolithotomy: a systematic review from the European association of urology urolithiasis guidelines panel. Eur Urol 72(2):220-235. https://doi.org/10.1016/j.eururo.2017.01. 046

4. Zeng G, Cai C, Duan X et al (2021) Mini percutaneous nephrolithotomy is a noninferior modality to standard percutaneous nephrolithotomy for the management of $20-40 \mathrm{~mm}$ renal calculi: a multicenter randomized controlled trial. Eur Urol 79(1):114-121. https:// doi.org/10.1016/j.eururo.2020.09.026

5. Thapa BB, Niranjan V (2020) Mini PCNL over standard PCNL: what makes it better? Surg J (New York) 6(1):e19-e23. https://doi. org/10.1055/s-0040-1701225

6. Wu C, Hua L-X, Zhang J-Z, Zhou X-R, Zhong W, Ni H-D (2017) Comparison of renal pelvic pressure and postoperative fever incidence between standard- and mini-tract percutaneous nephrolithotomy. Kaohsiung J Med Sci 33(1):36-43. https://doi. org/10.1016/j.kjms.2016.10.012

7. Haupt G, Pannek J, Herde T, Schulze H, Senge T (1995) The lithovac: new suction device for the Swiss lithoclast. J Endourol 9(5):375-377. https://doi.org/10.1089/end.1995.9.375

8. Tzelves L, Skolarikos A (2020) Suction use during endourological procedures. Curr Urol Rep 21(11):46. https://doi.org/10.1007/ s11934-020-00998-9

9. Shah D, Patil A, Reddy N et al (2020) A clinical experience of thulium fibre laser in miniperc to dust with suction: a new horizon. World J Urol. https://doi.org/10.1007/s00345-020-03458-8

10. Liu Y, Zhu W, Zeng G (2021) Percutaneous nephrolithotomy with suction: is this the future? Curr Opin Urol 31(2):95-101. https://doi. org/10.1097/MOU.0000000000000854

11. Zanetti SP, Boeri L, Gallioli A, Talso M, Montanari E (2017) Minimally invasive PCNL-MIP. Arch Esp Urol 70(1):226-234

12. Zanetti SP, Lievore E, Fontana M et al (2020) Vacuum-assisted mini-percutaneous nephrolithotomy: a new perspective in fragments clearance and intrarenal pressure control. World J Urol. https://doi. org/10.1007/s00345-020-03318-5

13. Lai D, Chen M, Sheng M et al (2020) Use of a novel vacuumassisted access sheath in minimally invasive percutaneous nephrolithotomy: a feasibility study. J Endourol 34(3):339-344. https://doi. org/10.1089/end.2019.0652

14. Lievore E, Boeri L, Zanetti SP et al (2020) Clinical comparison of mini-percutaneous nephrolithotomy with vacuum cleaner effect or with a vacuum-assisted access sheath: a single center experience. $\mathrm{J}$ Endourol. https://doi.org/10.1089/end.2020.0555

15. Charlson ME, Pompei P, Ales KL, MacKenzie CR (1987) A new method of classifying prognostic comorbidity in longitudinal studies: development and validation. J Chronic Dis 40(5):373-383. https://doi.org/10.1016/0021-9681(87)90171-8

16. Ito H, Kawahara T, Terao $\mathrm{H}$ et al (2012) The most reliable preoperative assessment of renal stone burden as a predictor of stone-free status after flexible ureteroscopy with holmium laser lithotripsy: a single-center experience. Urology 80(3):524-528. https://doi.org/ 10.1016/j.urology.2012.04.001

17. Bonkat G, Bartoletti R., Bruyere F, et al. (2021) EAU Guidelines on Urological Infections. Eur Assoc Urol. https://uroweb.org/wp-conte nt/uploads/EAU-Guidelines-on-Urological-infections-2021.pdf.

18. de la Rosette JJMCH, Opondo D, Daels FPJ et al (2012) Categorisation of complications and validation of the Clavien score for percutaneous nephrolithotomy. Eur Urol 62(2):246-255. https://doi. org/10.1016/j.eururo.2012.03.055

19. D'Agostino RB (1998) Propensity score methods for bias reduction in the comparison of a treatment to a non-randomized control group. Stat Med 17(19):2265-2281. https://doi.org/10.1002/(SICI)10970258(19981015)17:19\%3c2265::AID-SIM918\%3e3.0.CO;2-B

20. Zeng G, Wan S, Zhao Z et al (2016) Super-mini percutaneous nephrolithotomy (SMP): a new concept in technique and instrumentation. BJU Int 117(4):655-661. https://doi.org/10.1111/bju.13242

21. Zeng G, Zhu W, Liu Y, Fan J, Zhao Z, Cai C (2017) The new generation super-mini percutaneous nephrolithotomy (SMP) system: a step-by-step guide. BJU Int 120(5):735-738. https://doi.org/10. 1111/bju. 13955

22. Song L, Chen Z, Liu T et al (2011) The application of a patented system to minimally invasive percutaneous nephrolithotomy. J Endourol 25(8):1281-1286. https://doi.org/10.1089/end.2011.0032

23. Liu Y, Cai C, Aquino A et al (2020) Management of large renal stones with super-mini percutaneous nephrolithotomy: an international multicentre comparative study. BJU Int 126(1):168-176. https://doi.org/10.1111/bju.15066

24. Xu G, He Y, Zhao H et al (2016) Mini-nephroscope combined with pressure suction: an effective tool in MPCNL for intrarenal stones in patients with urinary tract infections. Urolithiasis 44(5):445-450. https://doi.org/10.1007/s00240-016-0859-8 
25. Huang J, Song L, Xie D et al (2016) A randomized study of minimally invasive percutaneous nephrolithotomy (MPCNL) with the aid of a patented suctioning sheath in the treatment of renal calculus complicated by pyonephrosis by one surgery. BMC Urol 16(1):71. https://doi.org/10.1186/s12894-016-0184-0

26. Bechis SK, Han DS, Abbott JE et al (2018) Outpatient percutaneous nephrolithotomy: the UC San Diego health experience. J Endourol 32(5):394-401
Publisher's Note Springer Nature remains neutral with regard to jurisdictional claims in published maps and institutional affiliations. 